\author{
${ }^{1}$ A.N. Stanzhitskii ${ }^{\text {iD }},{ }^{2}$ S.G. Karakenova ${ }^{\text {iD }},{ }^{3}$ S.S. Zhumatov \\ ${ }^{1}$ Doctor of Physical and Mathematical Sciences, E-mail: ostanzh@gmail.com, \\ Taras Shevchenko National University of Kyiv, Kyiv, Ukraine \\ ${ }^{2} \mathrm{PhD}$ student, E-mail: sayakhat.karakenova05@gmail.com, \\ Al-Farabi Kazakh National University, Almaty, Kazakhstan \\ ${ }^{3}$ Doctor of Physical and Mathematical Sciences, E-mail: sailau.math@gmail.com, \\ Institute of Mathematics and Mathematical Modeling, Almaty, Kazakhstan
}

\title{
ON A COMPARISON THEOREM FOR STOCHASTIC INTEGRO-FUNCTIONAL EQUATIONS OF NEUTRAL TYPE
}

In this paper, we will discuss a comparison result for solutions to the Cauchy problems for two stochastic differential equations with delay. On this subject number of authors have obtained their comparison results. We deal with the Cauchy problems for two integro-differential equations. Except transient- (or drift-) and diffusion coefficients our equations include also one integrodifferential term. Basic difference of our case from the case of all earlier investigated problems is presence of this term. We introduce a concept of solutions to our problems and prove the comparison theorem for them. According to our result under certain assumptions on coefficients of equations under consideration, their solutions depend on the transient-coefficients in a monotone way.

Key words: stochastic differential equation, comparison theorem, Hilbert space.

${ }^{1}$ А.Н. Станжицкий,${ }^{2}$ С.Г. Каракенова,${ }^{3}$ С.С. Жуматов

${ }^{1}$ ф.-м.ғ.д., Т. Шевченко атындағы Киев ұлттық университеті, Киев қ., Украина, E-mail: ostanzh@gmail.com

2 докторант, әл-Фараби атындағы Қазақ ұлттық университеті,

Алматы қ., Қазақстан, E-mail: sayakhat.karakenova05@gmail.com

${ }^{3}$ ф.-м.ғ.д., Математика және математикалық моделдеу институты

Алматы қ., Қазақстан, E-mail: sailau.math@gmail.com

Бейтарап типтегі стохастикалық интегралдық-функционалдық теңдеулер үшін салыстыру теоремасы

Бұл мақалада кідіріс әсерлі екі стохастикалық дифференциалдық теңдеулер үшін Коши есебі шешімдерін салыстыру есебі қарастырылған. Осы сияқты есептердің шешімдерін салыстыруға қатысты көптеген авторлар өз нәтижелерін алды. Мақалада бейтарап типті екі стохастикалық интегралдық-дифференциалдық теңдеулер үшін Коши есебі қарастырылған. Қарастырылатын есептің тасымалдау және диффузия коэффициенттерінен басқа бір интегралдық-дифференциалдық бөліктен тұрады. Бұрын зерттелген жұмыстардан негізгі айырмашылығы интегралдық бөліктің бар болуы болып табылады. Алынған нәтижелерге сәйкес, олардың шешімі тасымалдау коэффициентіне монотонды тәуелді болады.

Түйін сөздер: стохастикалық дифференциалдық теңдеу, салыстыру теоремасы, гильберт кеңістігі.

${ }^{1}$ А.Н. Станжицкий,${ }^{2}$ С.Г. Каракенова,${ }^{3}$ С.С. Жуматов

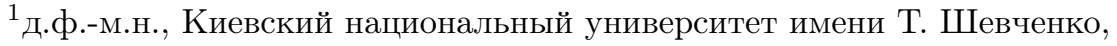

г. Киев, Украина, E-mail: ostanzh@gmail.com

2 докторант, Казахский национальный университет имени аль-Фараби,

г. Алматы, Казахстан, E-mail: sayakhat.karakenova05@gmail.com

3 д.ф.-м.н., Институт математики и математического моделирования, г. Алматы, Казахстан, E-mail: sailau.math@gmail.com

Об одной теореме сравнения для стохастических интегро-функциональных уравнений нейтрального типа 
В данной статье рассматривается задача сравнения решений задач Коши для двух стохастических дифференциальных уравнений с запаздыванием. В этой области множество авторов получили свои результаты, касающиеся сравнения решений подобных задач. В данной работе рассматриваются задачи Коши для двух стохастических интегродифференциальных уравнений нейтрального типа. Помимо коэффициента сноса (переноса) и коэффициента диффузии, рассматриваемые уравнения содержат также один интегродифференциальный член. Наличие этого интегрального члена является основным отличием этой задачи ото всех ранее исследуемых задач. Для наших задач вводятся понятия решений, для которых доказана теорема сравнения. Согласно полученному результату, при некоторых предположениях на коэффициенты рассматриваемых уравнений, их решения монотонно зависят от коэффициентов переноса

Ключевые слова: стохастическое дифференциальное уравнение, теорема сравнения, гильбертово пространство.

\section{Introduction}

In the given paper the following Cauchy problems for two neutral stochastic integrodifferential equations

$$
\begin{gathered}
d\left(u_{i}(t, x)+\int_{\mathbb{R}^{d}} b_{i}\left(t, x, u_{i}(\alpha(t), \xi), \xi\right) d \xi\right)=f_{i}\left(t, u_{i}(\alpha(t), x), x\right) d t \\
+\sigma(t, x) d \beta(t), 0<t \leq T, x \in \mathbb{R}^{d}, i \in\{1,2\}, \\
u_{i}(t, x)=\phi_{i}(t, x), \quad-r \leq t \leq 0, x \in \mathbb{R}^{d}, r>0, i \in\{1,2\},
\end{gathered}
$$

are studied, where $T>0$ is fixed, $\beta$ is one-dimensional Brownian motion, $f_{i}:[0, T] \times$ $\times \mathbb{R} \times \mathbb{R}^{d} \rightarrow \mathbb{R}, i \in\{1,2\}, \sigma:[0, T] \times \mathbb{R}^{d} \rightarrow \mathbb{R}$ and $b_{i}:[0, T] \times \mathbb{R}^{d} \times \mathbb{R} \times \mathbb{R}^{d} \rightarrow \mathbb{R}, i \in\{1,2\}$, are some given functions to be specified later, $\phi_{i}:[-r, 0] \times \mathbb{R}^{d} \rightarrow \mathbb{R}, i \in\{1,2\}$, are initial-datum functions, $\alpha:[0, T] \rightarrow[-r,+\infty)$ is a delay function. For solutions $u_{1}$ and $u_{2}$ of these problems a comparison theorem is proved. According to the obtained result, if $f_{1} \geq f_{2}$, then $u_{1} \geq u_{2}$ with probability one. A comparison problem for solutions to stochastic differential equations in finite-dimensional case has firstly arised in [14]. A comparison theorem for equation of the form $d \xi(t)=f(t, \xi(t)) d t+\sigma(t, \xi(t)) d \beta(t)$ has been proved in this work by A. V. Skorokhod. According to this theorem, under certain assumptions, a solution of the equation above is monotonously non-decreasing function, depending on drift-coefficient $f$. A more general presentation of the comparison theorem is given in [11], [13]. Variations of these results have been proposed in [2] - [10]. The aim of the given work was to prove the comparison theorem for solutions of problem $(1)-\left(1^{*}\right)$.

\section{Literature review}

For the first time, the problem of the comparison of solutions of stochastic equations in the finite-dimensional case arose in [14]. It is proved in it that, under certain assumptions, the solution of the equation is a monotone non-decreasing function of the transfer coefficient. A more general form of the comparison theorem is given in [10] [13]. Variations of these results were proposed in [1], [2], [3], [5], [7]-[9], [11]-[25]. Comparison theorems for solutions of stochastic partial differential equations with a multidimensional Wiener process are presented 
in [3]. In [6], a proof is presented of a comparison theorem for solutions of the Cauchy problem for stochastic differential equations with a multidimensional Wiener process in a Hilbert space. The aim of this paper was to prove a comparison theorem for solutions of problem (1) using ideas from [6] and [14]. This result plays an important role in studying the existence and uniqueness of a solution to this problem under non-Lipschitz conditions on drift coefficients.

The structure of the article is as follows: Section 2 contains the statement of the problem, 3 - preliminary facts and auxiliary results, 4 - proof of the main theorem.

\section{Material and methods}

\subsection{Comparison theorem for stochastic differential equations in the finite- dimensional case}

We consider the Cauchy problem of the form

$$
\begin{aligned}
& d\left(u_{i}(t, x)+\int_{\mathbb{R}^{d}} b_{i}\left(t, x, u_{i}(\alpha(t), \xi), \xi\right) d \xi\right)=f_{i}\left(t, u_{i}(\alpha(t), x), x\right) d t+\sigma(t, x) d \beta(t), \\
& 0<t \leq T, x \in \mathbb{R}^{d}, i \in\{1,2\}, \\
& u_{i}(t, x)=\phi_{i}(t, x),-r \leq t \leq 0, i \in\{1,2\},
\end{aligned}
$$

where $\beta$ one-dimensional Brownian motion. We regard the following conditions to be fulfilled:

1. $\alpha:[0, T] \rightarrow[-r,+\infty)$ belongs to $C^{1}([0, T])$ with $0<\alpha^{\prime}<1$.

2. $f_{i}:[0, T] \times \mathbb{R} \times \mathbb{R}^{d} \rightarrow \mathbb{R}, i \in\{1,2\}, \sigma:[0, T] \times \mathbb{R} \times \mathbb{R}^{d} \rightarrow[0, \infty], b:[0, T] \times \mathbb{R}^{d} \times \mathbb{R} \times \mathbb{R}^{d} \rightarrow$ $\rightarrow \mathbb{R}$ are measurable functions.

3. The initial-datum functions $\phi(t, x, \omega):[-r, 0] \times \mathbb{R}^{d} \times \Omega \rightarrow L_{2}\left(\mathbb{R}^{d}\right), i \in\{1,2\}$, are $\mathcal{F}_{0}$-measurable random variables and such that

$$
\mathbf{E} \sup _{-r \leq t \leq 0}\left\|\phi_{i}(t, \cdot)\right\|_{L_{2}\left(\mathbb{R}^{d}\right)}^{2}<\infty, i \in\{1,2\}, \quad \mathbf{E} \sup _{-r \leq t \leq 0} \phi^{2}(t, x)<\infty ; .
$$

4. $b$, satisfy the Lipshitz condition in the third argument of the form

$$
\begin{gathered}
|b(t, x, u, \xi)-b(t, x, \nu, \xi)| \leq l(t, x, \xi)|u-\nu| \\
0 \leq t \leq T,\{x, \xi\} \subset \mathbb{R}^{d},\{u, v\} \subset \mathbb{R},
\end{gathered}
$$

where the conditions are valid for the function $l$

$$
\sup _{0 \leq t \leq T} \int_{\mathbb{R}^{d}}\left(\int_{\mathbb{R}^{d}} \frac{l^{2}(t, x, \xi)}{\rho(\xi)} d \xi\right) \rho(x) d x<\frac{1}{4}, \sup _{0 \leq t \leq T} \int_{\mathbb{R}^{d}} \frac{l^{2}(t, x, \xi)}{\rho(\xi)} d \xi<\infty, x \in \mathbb{R}^{d} .
$$


5. There exists a function $b_{1}: \mathbb{R}^{d} \times \mathbb{R}^{d} \rightarrow[0, \infty)$, such that

$$
\int_{\mathbb{R}^{d}}\left(\int_{\mathbb{R}^{d}} b_{1}(x, \xi) d \xi\right)^{2} \rho(x) d x<\infty, \quad \quad \int_{\mathbb{R}^{d}} b_{1}(x, \xi) d \xi<\infty, x \in \mathbb{R}^{d} ;
$$

such that

$$
\sup _{0 \leq t \leq T}|b(t, x, 0, \xi)| \leq b_{1}(x, \xi), x \in \mathbb{R}^{d}, \xi \in \mathbb{R}^{d}
$$

6. The function $f_{i}, \sigma, i \in 1,2$, satisfy the conditions of linear growth and Lipschitz in the second argument, that is, there are $L>0$ such that

$$
\left|f_{i}(t, u, x)\right|+|\sigma(t, u, x)| \leq L(1+|u|), 0 \leq t \leq T, u \in \mathbb{R}, x \in \mathbb{R}^{d},
$$

$\left|f_{i}(t, u, x)-f_{i}(t, \nu, x)\right|+|\sigma(t, u, x)-| \sigma(t, \nu, x)|\leq L| u-\nu \mid, 0 \leq t \leq T,\{u, \nu\} \in \mathbb{R}, x \in \mathbb{R}^{d}, i \in 1,2$.

Let $u \equiv u_{i}, \phi \equiv \phi_{i}, b \equiv b_{i}, f \equiv f_{i}, i \in\{1,2\}$.

Definition. A continuous random process $u_{i}(t, \cdot, \omega):[-r, T] \times \Omega \rightarrow \mathbb{R}, i \in\{1,2\}$ is called a solution to $(1)-\left(1^{*}\right)$ provided

1. It is $\mathcal{F}_{t}$-measurable for almost all $-r \leq t \leq T$.

2. It satisfies the following integral equation

$$
\begin{aligned}
u_{i}(t, \cdot) & =\phi(0, \cdot)+\int_{\mathbb{R}^{d}} b(0, \cdot, \phi(-r, \xi), \xi) d \xi-\int_{\mathbb{R}^{d}} b\left(t, \cdot, u_{i}(\alpha(t), \xi), \xi\right) d \xi \\
& +\int_{0}^{t} f_{i}\left(s, u_{i}(\alpha(s), \cdot), \cdot\right) d s+\int_{0}^{t} \sigma\left(s, u_{i}(\alpha(s), \cdot), \cdot\right) d \beta(s), 0 \leq t \leq T, i \in\{1,2\}, \\
u_{i}(t, \cdot) & =\phi(t,), \quad-r \leq t \leq 0, i \in\{1,2\} .
\end{aligned}
$$

3. It satisfies the condition

$$
\mathbf{E} \int_{0}^{T}\left\|u_{i}(t, \cdot)\right\|_{L_{2}\left(\mathbb{R}^{d}\right)}^{2} d t<\infty, \quad \mathbf{E} \int_{0}^{T} u_{i}{ }^{2}(t, \cdot) d t<\infty, i \in\{1,2\} .
$$

The following theorems are true.

Theorem 1. Denote by $u=u_{i}, f=f_{i}, i \in 1,2$. Assume that the conditions (1)-(6) are satisfied. Then (7) has a solution continuous with probability one, unique in the sense that if 
$u(t, \cdot), \nu(t, \cdot), 0<t<T$ are two continuous solutions to $(7)$, then $\mathbf{P}\left\{\sup _{0 \leq t \leq T}|u(t, \cdot)-\nu(t, \cdot)|>\right.$ $0\}=0$.

Theorem 2. Suppose that conditions (1)-(6) are satisfied. Suppose further that $u_{i}(t, x), 0<t<T, i \in\{1,2\}$ are continuous (with probability one) solutions to problem (7). That, if $f_{1}(t, u, \cdot) \leq f_{2}(t, u, \cdot)$ for all $0 \leq t \leq T$ the condition $u_{1}(t, x) \leq u_{2}(t, x)$ are satisfied.

Proof of the theorem 1. In order to prove existence and uniqueness of solution to (7) we use the method of successive approximations. The idea of the proof is to construct a sequence of approximations, which converges to the solution $u$. From now on $x$ is supposed to be fixed. Let

$$
\begin{aligned}
& u^{(0)}(t, \cdot)=\phi(0, \cdot), 0<t \leq T, \\
& u^{(0)}(t, \cdot)=\phi(t, \cdot),-r \leq t \leq 0,
\end{aligned}
$$

and for $n \in\{1,2, \ldots\}$ define $u^{(n)}$ as

$$
\begin{aligned}
& u^{(n)}(t, \cdot)=\phi(0, \cdot)+\int_{\mathbb{R}^{d}} b(0, \cdot, \phi(-r, \xi), \xi) d \xi-\int_{\mathbb{R}^{d}} b\left(t, \cdot, u^{(n-1)}(\alpha(t), \xi), \xi\right) d \xi \\
&+\int_{0}^{t} f\left(s, u^{(n-1)}(\alpha(s), \cdot), \cdot\right) d s+\int_{0}^{t} \sigma(s, \cdot) d \beta(s), 0<t \leq T, \\
& u^{(n)}(t, \cdot)=\phi(t, \cdot),-r \leq t \leq 0 .
\end{aligned}
$$

1.1 Firstly let us choose a small $0 \leq T_{1} \leq T$ and prove that $\sup _{0 \leq t \leq T_{1}} \mathbf{E}\left\|u^{(n)}(t, \cdot)\right\|_{L_{2}\left(\mathbb{R}^{d}\right)}^{2}$ has a bound, independent of $n$. We obtain

$$
\begin{aligned}
& \sup _{0 \leq t \leq T_{1}} \mathbf{E}\left\|u^{(n)}(t, \cdot)\right\|_{L_{2}\left(\mathbb{R}^{d}\right)}^{2} \leq 8 \mathbf{E}\|\phi(0, \cdot)\|_{L_{2}\left(\mathbb{R}^{d}\right)}^{2}+8 \mathbf{E}\left\|\int_{\mathbb{R}^{d}}|b(0, \cdot, \phi(-r, \xi), \xi)| d \xi\right\|_{L_{2}\left(\mathbb{R}^{d}\right)}^{2} \\
& \quad+2 \sup _{0 \leq t \leq T_{1}} \mathbf{E}\left\|\int_{\mathbb{R}^{d}}\left|b\left(t, \cdot, u^{(n-1)}(\alpha(t), \xi), \xi\right)\right| d \xi\right\|_{L_{2}\left(\mathbb{R}^{d}\right)} \\
& \quad+8 \sup _{0 \leq t \leq T_{1}} \mathbf{E}\left\|\int_{0}^{t}\left|f\left(s, u^{(n-1)}(\alpha(s), \cdot), \cdot\right)\right| d s\right\|_{L_{2}\left(\mathbb{R}^{d}\right)}^{2}+8 \sup _{0 \leq t \leq T_{1}} \mathbf{E}\left\|\int_{0}^{t} \sigma(s, \cdot) d \beta(s)\right\|_{L_{2}\left(\mathbb{R}^{d}\right)}^{2} \\
& \quad=8 \mathbf{E}\|\phi(0, \cdot)\|_{L_{2}\left(\mathbb{R}^{d}\right)}^{2}+\sum_{j=1}^{4} S_{j}, 0<t \leq T
\end{aligned}
$$

From (2) and (4) we have

$$
\begin{gathered}
|b(t, \cdot, u, \xi)| \leq|b(t, \cdot, u, \xi)-b(t, \cdot, 0, \xi)|+|b(t, \cdot, 0, \xi)| \leq l(t, \cdot, \xi)|u|+\chi(\cdot, \xi), \\
0 \leq t \leq T, u \in \mathbb{R}, \xi \in \mathbb{R}^{d} .
\end{gathered}
$$


Then we obtain

$$
\begin{aligned}
S_{1}= & 8 \mathbf{E} \int_{\mathbb{R}^{d}}\left(\int_{\mathbb{R}^{d}}|b(0, x, \phi(-r, \xi), \xi)| d \xi\right)^{2} d x \leq 16 \mathbf{E} \int_{\mathbb{R}^{d}}\left(\int_{\mathbb{R}^{d}} l(0, x, \xi) \phi(-r, \xi) d \xi\right)^{2} d x \\
& +16 \int_{\mathbb{R}^{d}}\left(\int_{\mathbb{R}^{d}} \chi(x, \xi) d \xi\right)^{2} d x \leq 16\left(\int_{\mathbb{R}^{d}} \int_{\mathbb{R}^{d}} l^{2}(0, x, \xi) d \xi d x\right) \mathbf{E}\|\phi(-r, \cdot)\|_{L_{2}\left(\mathbb{R}^{d}\right)}^{2} \\
& +16 \int_{\mathbb{R}^{d}}\left(\int_{\mathbb{R}^{d}} \chi(x, \xi) d \xi\right)^{2} d x, \\
S_{2}= & 2 \sup _{0 \leq t \leq T_{1}} \mathbf{E} \int_{\mathbb{R}^{d}}\left(\int_{\mathbb{R}^{d}}\left|b\left(t, x, u^{(n-1)}(\alpha(t), \xi), \xi\right)\right| d \xi\right)^{2} d x \leq 4\left(\sup _{0 \leq t \leq T} \int_{\mathbb{R}^{d}} \int_{\mathbb{R}^{d}} l^{2}(t, x, \xi) d \xi d x\right) \\
& \times \sup _{0 \leq t \leq T_{1}} \mathbf{E}\left\|u^{(n-1)}(\alpha(t), \cdot)\right\|_{L_{2}\left(\mathbb{R}^{d}\right)}^{2}+4 \int_{\mathbb{R}^{d}}\left(\int_{\mathbb{R}^{d}} \chi(x, \xi) d \xi\right)^{2} d x .
\end{aligned}
$$

According to properties of $\alpha$, there exists a point $0 \leq t^{*} \leq T_{1}, \alpha\left(t^{*}\right)=0$. Then

$$
\begin{aligned}
& \sup _{0 \leq t \leq T_{1}} \mathbf{E}\left\|u^{(n-1)}(\alpha(t), \cdot)\right\|_{L_{2}\left(\mathbb{R}^{d}\right)}^{2} \leq \sup _{0 \leq t \leq t^{*}} \mathbf{E}\left\|u^{(n-1)}(\alpha(t), \cdot)\right\|_{L_{2}\left(\mathbb{R}^{d}\right)}^{2} \\
& +\sup _{t^{*} \leq t \leq \alpha\left(T_{1}\right)} \mathbf{E}\left\|u^{(n-1)}(\alpha(t), \cdot)\right\|_{L_{2}\left(\mathbb{R}^{d}\right)}^{2} \leq \sup _{-r \leq t \leq 0} \mathbf{E}\|\phi(t, \cdot)\|_{L_{2}\left(\mathbb{R}^{d}\right)}^{2}+\sup _{0 \leq t \leq T_{1}} \mathbf{E}\left\|u^{(n-1)}(t, \cdot)\right\|_{L_{2}\left(\mathbb{R}^{d}\right)}^{2}
\end{aligned}
$$

and we get from (11)

$$
\begin{aligned}
S_{2} & \leq 4\left(\sup _{0 \leq t \leq T} \int_{\mathbb{R}^{d}} \int_{\mathbb{R}^{d}} l^{2}(t, x, \xi) d \xi d x\right)\left(\sup _{-r \leq t \leq 0} \mathbf{E}\|\phi(t, \cdot)\|_{L_{2}\left(\mathbb{R}^{d}\right)}^{2}+\sup _{0 \leq t \leq T_{1}} \mathbf{E}\left\|u^{(n-1)}(t, \cdot)\right\|_{L_{2}\left(\mathbb{R}^{d}\right)}^{2}\right) \\
& +4 \int_{\mathbb{R}^{d}}\left(\int_{\mathbb{R}^{d}} \chi(x, \xi) d \xi\right)^{2} d x .
\end{aligned}
$$

If $t^{*}$ does not exist, then $\alpha(t)<0$ for all $t$ and further conclusions are obvious, because

$$
\sup _{0 \leq t \leq T_{1}} \mathbf{E}\left\|u^{(n-1)}(\alpha(t), \cdot)\right\|_{L_{2}\left(\mathbb{R}^{d}\right)}^{2}=\sup _{-r \leq t \leq 0} \mathbf{E}\|\phi(t, \cdot)\|_{L_{2}\left(\mathbb{R}^{d}\right)}^{2} \cdot
$$

In order to estimate $S_{3}$, we take (5) into account and obtain

$$
S_{3}=8 \sup _{0 \leq t \leq T_{1}} \mathbf{E} \int_{\mathbb{R}^{d}}\left(\int_{0}^{t}\left|f\left(s, u^{(n-1)}(\alpha(s), x), x\right)\right| d s\right)^{2} d x \leq 16 T_{1} \sup _{0 \leq t \leq T_{1}} \mathbf{E} \int_{0}^{t} \int_{\mathbb{R}^{d}}\left(\eta^{2}(s, x)\right.
$$


$\left.+L^{2}\left(u^{(n-1)}(\alpha(s), x)\right)^{2}\right) d x d s \leq 16 T_{1}\left(T_{1} \sup _{0 \leq t \leq T_{1}} \int_{\mathbb{R}^{d}} \eta^{2}(t, x) d x+L^{2} \int_{-r}^{\alpha\left(T_{1}\right)} \mathbf{E}\left\|u^{(n-1)}(s, \cdot)\right\|_{L_{2}\left(\mathbb{R}^{d}\right)}^{2} d s\right)$ $\leq 16 T_{1}^{2} \sup _{0 \leq t \leq T_{1}} \int_{\mathbb{R}^{d}} \eta^{2}(t, x) d x+16 L^{2} T_{1}\left(r \sup _{-r \leq s \leq 0} \mathbf{E}\|\phi(s, \cdot)\|_{L_{2}\left(\mathbb{R}^{d}\right)}^{2}+\int_{0}^{T_{1}} \mathbf{E}\left\|u^{(n-1)}(s, \cdot)\right\|_{L_{2}\left(\mathbb{R}^{d}\right)}^{2} d s\right)$.

For $S_{4}$ we conclude

$$
S_{4}=8 \sup _{0 \leq t \leq T_{1}} \int_{\mathbb{R}^{d}} \int_{0}^{t}\left(\sigma^{2}(s, x) d s\right) d x \leq 8 \int_{0}^{T_{1}}\|\sigma(s, \cdot)\|_{L_{2}\left(\mathbb{R}^{d}\right)}^{2} d s .
$$

Let denote

$$
\begin{gathered}
S\left(T_{1}\right)=8 \mathbf{E}\|\phi(0, \cdot)\|_{L_{2}\left(\mathbb{R}^{d}\right)}^{2}+16\left(\int_{\mathbb{R}^{d}} \int_{\mathbb{R}^{d}} l^{2}(0, x, \xi) d \xi d x\right) \mathbf{E}\|\phi(-r, \cdot)\|_{L_{2}\left(\mathbb{R}^{d}\right)}^{2} \\
+20 \iint_{\mathbb{R}^{d}}\left(\int_{\mathbb{R}^{d}} \chi(x, \xi) d \xi\right)^{2} d x+4\left(\sup _{0 \leq t \leq T} \int_{\mathbb{R}^{d}} \int_{\mathbb{R}^{d}} l^{2}(t, x, \xi) d \xi d x\right) \sup _{-r \leq t \leq 0} \mathbf{E}\|\phi(t, \cdot)\|_{L_{2}\left(\mathbb{R}^{d}\right)}^{2} \\
+16 T_{1}^{2} \sup _{0 \leq t \leq T_{1}} \int_{\mathbb{R}^{d}} \eta^{2}(t, x) d x+16 r L^{2} T_{1} \sup _{-r \leq t \leq 0} \mathbf{E}\|\phi(t, \cdot)\|_{L_{2}\left(\mathbb{R}^{d}\right)}^{2}+8 \int_{0}^{T_{1}}\|\sigma(t, \cdot)\|_{L_{2}\left(\mathbb{R}^{d}\right)}^{2} d t<\infty .
\end{gathered}
$$

Then from (10) we obtain

$$
\begin{array}{rl}
\sup _{0 \leq t \leq T_{1}} & \mathbf{E}\left\|u^{(n)}(t, \cdot)\right\|_{L_{2}\left(\mathbb{R}^{d}\right)}^{2} \leq S\left(T_{1}\right)+4\left(\sup _{0 \leq t \leq T} \int_{\mathbb{R}^{d}} \int_{\mathbb{R}^{d}} l^{2}(t, x, \xi) d \xi d x\right) \\
& \times \sup _{0 \leq t \leq T_{1}} \mathbf{E}\left\|u^{(n-1)}(t, \cdot)\right\|_{L_{2}\left(\mathbb{R}^{d}\right)}^{2}+16 L^{2} T_{1} \int_{0}^{T_{1}} \mathbf{E}\left\|u^{(n-1)}(t, \cdot)\right\|_{L_{2}\left(\mathbb{R}^{d}\right)}^{2} d t .
\end{array}
$$

If $n=1$, then from (12) we have

$$
\begin{aligned}
& \sup _{0 \leq t \leq T_{1}} \mathbf{E}\left\|u^{(1)}(t, \cdot)\right\|_{L_{2}\left(\mathbb{R}^{d}\right)}^{2} \leq S\left(T_{1}\right)+4\left(\sup _{0 \leq t \leq T} \int_{\mathbb{R}^{d}} \int_{\mathbb{R}^{d}} l^{2}(t, x, \xi) d \xi d x\right) \mathbf{E}\|\phi(0, \cdot)\|_{L_{2}\left(\mathbb{R}^{d}\right)}^{2} \\
& \quad+16 L^{2} T_{1} \int_{0}^{T_{1}} \mathbf{E}\|\phi(0, \cdot)\|_{L_{2}\left(\mathbb{R}^{d}\right)}^{2} d t .
\end{aligned}
$$

For an arbitrary $n \in\{2,3, \ldots\}$ we obtain

$$
\sup _{0 \leq t \leq T_{1}} \mathbf{E}\left\|u^{(n)}(t, \cdot)\right\|_{L_{2}\left(\mathbb{R}^{d}\right)}^{2} \leq S\left(T_{1}\right)\left[1+4 \sup _{0 \leq t \leq T} \int_{\mathbb{R}^{d}} \int_{\mathbb{R}^{d}} l^{2}(t, x, \xi) d \xi d x+\ldots\right.
$$




$$
\begin{aligned}
& \left.+\left(4 \sup _{0 \leq t \leq T} \int_{\mathbb{R}^{d}} \int_{\mathbb{R}^{d}} l^{2}(t, x, \xi) d \xi d x\right)^{n-1}\right]+16 L^{2} T_{1} \int_{0}^{T_{1}} S\left(T_{1}\right)\left[1+4 \sup _{0 \leq t \leq T} \int_{\mathbb{R}^{d}} \int_{\mathbb{R}^{d}} l^{2}(t, x, \xi) d \xi d x\right. \\
& \left.+\ldots+\left(4 \sup _{0 \leq t \leq T} \int_{\mathbb{R}^{d}} \int_{\mathbb{R}^{d}} l^{2}(t, x, \xi) d \xi d x\right)^{n-2}\right] d s+16 L^{2} T_{1} \int_{0}^{T_{1}}\left(16 L^{2} T_{1}\left(T_{1}-s\right)\right) S\left(T_{1}\right) \\
& \times\left[1+\ldots+\left(4 \sup _{0 \leq t \leq T} \int_{\mathbb{R}^{d}} \int_{\mathbb{R}^{d}} l^{2}(t, x, \xi) d \xi d x\right)^{n-3}\right] d s \\
& +\ldots+16 L^{2} T_{1} \int_{0}^{T_{1}} \frac{\left(16 L^{2} T_{1}\left(T_{1}-s\right)\right)^{n-3}}{(n-3) !} S\left(T_{1}\right)\left[1+4 \sup _{0 \leq t \leq T} \int_{\mathbb{R}^{d}} \int_{\mathbb{R}^{d}} l^{2}(t, x, \xi) d \xi d x\right] d s \\
& +\left(4 \sup _{0 \leq t \leq T} \int_{\mathbb{R}^{d}} \int_{\mathbb{R}^{d}} l^{2}(t, x, \xi) d \xi d x\right)^{n-1}\left[\left(4 \sup _{0 \leq t \leq T} \int_{\mathbb{R}^{d}} \int_{\mathbb{R}^{d}} l^{2}(t, x, \xi) d \xi d x\right) \mathbf{E}\|\phi(0, \cdot)\|_{L_{2}\left(\mathbb{R}^{d}\right)}^{2}\right. \\
& \left.+16 L^{2} T_{1} \int_{0}^{T_{1}} \mathbf{E}\|\phi(0, \cdot)\|_{L_{2}\left(\mathbb{R}^{d}\right)}^{2} d s\right]+16 L^{2} T_{1}\left(4 \sup _{0 \leq t \leq T} \int_{\mathbb{R}^{d}} \int_{\mathbb{R}^{d}} l^{2}(t, x, \xi) d \xi d x\right)^{n-2} \\
& \times \int_{0}^{T_{1}}\left[\left(4 \sup _{0 \leq t \leq T} \int_{\mathbb{R}^{d}} \int_{\mathbb{R}^{d}} l^{2}(t, x, \xi) d \xi d x\right) \mathbf{E}\|\phi(0, \cdot)\|_{L_{2}\left(\mathbb{R}^{d}\right)}^{2}+16 L^{2} T_{1} \int_{0}^{T_{1}} \mathbf{E}\|\phi(0, \cdot)\|_{L_{2}\left(\mathbb{R}^{d}\right)}^{2} d s\right] d \tau \\
& \left(16 L^{2} T_{1}\right)^{2}\left(4 \sup _{0 \leq t \leq T} \int_{\mathbb{R}^{d}} \int_{\mathbb{R}^{d}} l^{2}(t, x, \xi) d \xi d x\right)^{n-3} \int_{0}^{T_{1}}\left(T_{1}-\tau\right) \\
& \times\left[\left(4 \sup _{0 \leq t \leq T} \int_{\mathbb{R}^{d}} \int_{\mathbb{R}^{d}} l^{2}(t, x, \xi) d \xi d x\right) \mathbf{E}\|\phi(0, \cdot)\|_{L_{2}\left(\mathbb{R}^{d}\right)}^{2}+16 L^{2} T_{1} \int_{0}^{T_{1}} \mathbf{E}\|\phi(0, \cdot)\|_{L_{2}\left(\mathbb{R}^{d}\right)}^{2} d s\right] d \tau \\
& +\ldots+\left(16 L^{2} T_{1}\right)^{n-3}\left(4 \sup _{0 \leq t \leq T} \int_{\mathbb{R}^{d}} \int_{\mathbb{R}^{d}} l^{2}(t, x, \xi) d \xi d x\right)^{2} \int_{0}^{T_{1}} \frac{\left(T_{1}-\tau\right)^{n-4}}{(n-4) !} \\
& \times\left[\left(4 \sup _{0 \leq t \leq T} \int_{\mathbb{R}^{d}} \int_{\mathbb{R}^{d}} l^{2}(t, x, \xi) d \xi d x\right) \mathbf{E}\|\phi(0, \cdot)\|_{L_{2}\left(\mathbb{R}^{d}\right)}^{2}+16 L^{2} T_{1} \int_{0}^{T_{1}} \mathbf{E}\|\phi(0, \cdot)\|_{L_{2}\left(\mathbb{R}^{d}\right)}^{2} d s\right] d \tau \\
& +\left(16 L^{2} T_{1}\right)^{n-2}\left(4 \sup _{0 \leq t \leq T} \int_{\mathbb{R}^{d}} \int_{\mathbb{R}^{d}} l^{2}(t, x, \xi) d \xi d x\right) \int_{0}^{T_{1}} \frac{\left(T_{1}-\tau\right)^{n-3}}{(n-3) !} \\
& \times\left[\left(4 \sup _{0 \leq t \leq T} \int_{\mathbb{R}^{d}} \int_{\mathbb{R}^{d}} l^{2}(t, x, \xi) d \xi d x\right) \mathbf{E}\|\phi(0, \cdot)\|_{L_{2}\left(\mathbb{R}^{d}\right)}^{2}+16 L^{2} T_{1} \int_{0}^{T_{1}} \mathbf{E}\|\phi(0, \cdot)\|_{L_{2}\left(\mathbb{R}^{d}\right)}^{2} d s\right] d \tau
\end{aligned}
$$




$$
\begin{aligned}
& +\left(4 \sup _{0 \leq t \leq T} \int_{\mathbb{R}^{d}} \int_{\mathbb{R}^{d}} l^{2}(t, x, \xi) d \xi d x\right)^{n-2} 16 L^{2} T_{1} \int_{0}^{T_{1}} C\left(T_{1}\right) d s+\left(4 \sup _{0 \leq t \leq T} \int_{\mathbb{R}^{d}} \int_{\mathbb{R}^{d}} l^{2}(t, x, \xi) d \xi d x\right)^{n-3} \\
& \times 16 L^{2} T_{1} \int_{0}^{T_{1}}\left(16 L^{2} T_{1}\left(T_{1}-s\right)\right) C\left(T_{1}\right) d s+\ldots+\left(4 \sup _{0 \leq t \leq T} \int_{\mathbb{R}^{d}} \int_{\mathbb{R}^{d}} l^{2}(t, x, \xi) d \xi d x\right)^{2} 16 L^{2} T_{1} \\
& \times \int_{0}^{T_{1}} \frac{\left(16 L^{2} T_{1}\left(T_{1}-s\right)\right)^{n-4}}{(n-4) !} C\left(T_{1}\right) d s+\left(4 \sup _{0 \leq t \leq T} \int_{\mathbb{R}^{d}} \int_{\mathbb{R}^{d}} l^{2}(t, x, \xi) d \xi d x\right) 16 L^{2} T_{1} \\
& \times \int_{0}^{T_{1}} \frac{\left(16 L^{2} T_{1}\left(T_{1}-s\right)\right)^{n-3}}{(n-3) !} C\left(T_{1}\right) d s+16 L^{2} T_{1} \int_{0}^{T_{1}} \frac{\left(16 L^{2} T_{1}\left(T_{1}-s\right)\right)^{n-2}}{(n-2) !} C\left(T_{1}\right) d s \\
& +\left(4 \sup _{0 \leq t \leq T} \int_{\mathbb{R}^{d}} \int_{\mathbb{R}^{d}} l^{2}(t, x, \xi) d \xi d x\right)^{n-2} 16 L^{2} T_{1} \int_{0}^{T_{1}}\left(16 L^{2} T_{1}\left(T_{1}-s\right)\right) \mathbf{E}\|\phi(0, \cdot)\|_{L_{2}\left(\mathbb{R}^{d}\right)}^{2} d s \\
& +\left(4 \sup _{0 \leq t \leq T} \int_{\mathbb{R}^{d}} \int_{\mathbb{R}^{d}} l^{2}(t, x, \xi) d \xi d x\right)^{n-3} 16 L^{2} T_{1} \int_{0}^{T_{1}} \frac{\left(16 L^{2} T_{1}\left(T_{1}-s\right)\right)^{2}}{2} \mathbf{E}\|\phi(0, \cdot)\|_{L_{2}\left(\mathbb{R}^{d}\right)}^{2} d s \\
& +\ldots+\left(4 \sup _{0 \leq t \leq T} \int_{\mathbb{R}^{d}} \int_{\mathbb{R}^{d}} l^{2}(t, x, \xi) d \xi d x\right)^{2} 16 L^{2} T_{1} \int_{0}^{T_{1}} \frac{\left(16 L^{2} T_{1}\left(T_{1}-s\right)\right)^{n-3}}{(n-3) !} \mathbf{E}\|\phi(0, \cdot)\|_{L_{2}\left(\mathbb{R}^{d}\right)}^{2} d s \\
& +\left(4 \sup _{0 \leq t \leq T} \int_{\mathbb{R}^{d}} \int_{\mathbb{R}^{d}} l^{2}(t, x, \xi) d \xi d x\right) 16 L^{2} T_{1} \int_{0}^{T_{1}} \frac{\left(16 L^{2} T_{1}\left(T_{1}-s\right)\right)^{n-2}}{(n-2) !} \mathbf{E}\|\phi(0, \cdot)\|_{L_{2}\left(\mathbb{R}^{d}\right)}^{2} d s \\
& +16 L^{2} T_{1} \int_{0}^{T_{1}} \frac{\left(16 L^{2} T_{1}\left(T_{1}-s\right)\right)^{n-1}}{(n-1) !} \mathbf{E}\|\phi(0, \cdot)\|_{L_{2}\left(\mathbb{R}^{d}\right)}^{2} d s
\end{aligned}
$$

where $C\left(T_{1}\right)=S\left(T_{1}\right)+\left(4 \sup _{0 \leq t \leq T_{\mathbb{R}^{d}}} \iint_{\mathbb{R}^{d}} l^{2}(t, x, \xi) d \xi d x\right) \mathbf{E}\|\phi(0, \cdot)\|_{L_{2}\left(\mathbb{R}^{d}\right)}^{2}$. It is easy to see that if $T_{1}$ is small enough and assumption (3) is true, then the the right-hand of (13) is not more 
than

$$
\begin{gathered}
\frac{S\left(T_{1}\right)}{1-4 \sup _{0 \leq t \leq T_{\mathbb{R}^{d}}} \int_{\mathbb{R}^{d}} l^{2}(t, x, \xi) d \xi d x}+\frac{16 L^{2} T_{1} \cdot S\left(T_{1}\right) \cdot \int_{0}^{T_{1}} \exp \left\{16 L^{2} T_{1}\left(T_{1}-s\right)\right\} d s}{1-4 \sup _{0 \leq t \leq T_{\mathbb{R}^{d}}} \int_{\mathbb{R}^{d}} l^{2}(t, x, \xi) d \xi d x} \\
+\frac{C\left(T_{1}\right) \cdot \exp \left\{16 L^{2} T_{1}^{2}\right\}}{1-4 \sup _{0 \leq t \leq T} \iint_{\mathbb{R}^{d}} l_{\mathbb{R}^{d}}(t, x, \xi) d \xi d x}+\frac{16 L^{2} T_{1} \cdot \int_{0}^{T_{1}} \exp \left\{16 L^{2} T_{1}\left(T_{1}-s\right)\right\} d s}{1-4 \sup _{0 \leq t \leq T} \iint_{\mathbb{R}^{d}} l_{\mathbb{R}^{d}}(t, x, \xi) d \xi d x} \mathbf{E}\|\phi(0, \cdot)\|_{L_{2}\left(\mathbb{R}^{d}\right)}^{2} \\
=\frac{\left(S\left(T_{1}\right)+C\left(T_{1}\right)\right) \cdot \exp \left\{16 L^{2} T_{1}^{2}\right\}+\left(\exp \left\{16 L^{2} T_{1}^{2}\right\}-1\right) \mathbf{E}\|\phi(0, \cdot)\|_{L_{2}\left(\mathbb{R}^{d}\right)}^{2}}{1-4 \sup _{0 \leq t \leq T_{\mathbb{R}^{d}} \int} \int_{\mathbb{R}^{d}} l^{2}(t, x, \xi) d \xi d x} .
\end{gathered}
$$

Thus there exists $c\left(T_{1}\right)>0$ such that for an arbitrary $n \in\{1,2, \ldots\}$

$$
\sup _{0 \leq t \leq T_{1}} \mathbf{E}\left\|u^{(n)}(t, \cdot)\right\|_{L_{2}\left(\mathbb{R}^{d}\right)}^{2} \leq c\left(T_{1}\right)
$$

1.2 Second let us prove that $\left(u^{(n)}(t, \cdot), n \in\{1,2, \ldots\}\right), 0<t \leq T_{1}$, is convergent. In order to do it we estimate $\sup _{0 \leq t \leq T_{1}} \mathbf{E}\left\|u^{(n+1)}(t, \cdot)-u^{(n)}(t, \cdot)\right\|_{L_{2}\left(\mathbb{R}^{d}\right)}^{2}, n \in\{0,1, \ldots\}$.

If $n=0$, then we obtain, taking into account estimate (14),

$$
\begin{aligned}
& \sup _{0 \leq t \leq T_{1}} \mathbf{E}\left\|u^{(1)}(t, \cdot)-u^{(0)}(t, \cdot)\right\|_{L_{2}\left(\mathbb{R}^{d}\right)}^{2} \leq 2 \sup _{0 \leq t \leq T_{1}} \mathbf{E}\left\|u^{(1)}(t, \cdot)\right\|_{L_{2}\left(\mathbb{R}^{d}\right)}^{2} \\
& +2 \mathbf{E}\|\phi(0, \cdot)\|_{L_{2}\left(\mathbb{R}^{d}\right)}^{2}<\infty .
\end{aligned}
$$

If $n \in\{1,2, \ldots\}$, then we obtain, taking into account estimates from 1.1,

$$
\begin{aligned}
& \sup _{0 \leq t \leq T_{1}} \mathbf{E}\left\|u^{(n+1)}(t, \cdot)-u^{(n)}(t, \cdot)\right\|_{L_{2}\left(\mathbb{R}^{d}\right)}^{2} \leq 2\left(\sup _{0 \leq t \leq T} \int_{\mathbb{R}^{d}} \int_{\mathbb{R}^{d}} l^{2}(t, x, \xi) d \xi d x\right) \\
& \times \sup _{0 \leq t \leq T_{1}} \mathbf{E}\left\|u^{(n-1)}(t, \cdot)-u^{(n)}(t, \cdot)\right\|_{L_{2}\left(\mathbb{R}^{d}\right)}^{2}+2 L^{2} T_{1} \int_{0}^{T_{1}} \mathbf{E}\left\|u^{(n-1)}(s, \cdot)-u^{(n)}(s, \cdot)\right\|_{L_{2}\left(\mathbb{R}^{d}\right)}^{2} d s \\
& \leq\left(2 \sup _{0 \leq t \leq T} \iint_{\mathbb{R}^{d}} l_{\mathbb{R}^{d}}(t, x, \xi) d \xi d x+2 L^{2} T_{1}^{2}\right) \sup _{0 \leq t \leq T_{1}} \mathbf{E}\left\|u^{(n-1)}(t, \cdot)-u^{(n)}(t, \cdot)\right\|_{L_{2}\left(\mathbb{R}^{d}\right)}^{2} \leq \ldots \\
& \leq\left(2 \sup _{0 \leq t \leq T} \int_{\mathbb{R}^{d}} \int_{\mathbb{R}^{d}} l^{2}(t, x, \xi) d \xi d x+2 L^{2} T_{1}^{2}\right)^{n} \sup _{0 \leq t \leq T_{1}} \mathbf{E}\left\|u^{(0)}(t, \cdot)-u^{(1)}(t, \cdot)\right\|_{L_{2}\left(\mathbb{R}^{d}\right)}^{2} .
\end{aligned}
$$

Due to assumption (3) and choose of small $T_{1}, \sup _{0 \leq t \leq T_{\mathbb{R}^{d}}} \int_{\mathbb{R}^{d}} l^{2}(t, x, \xi) d \xi d x+L^{2} T_{1}^{2}<\frac{1}{2}$, 
therefore $\left(2 \sup _{0 \leq t \leq T_{\mathbb{R}^{d}}} \iint_{\mathbb{R}^{d}} l^{2}(t, x, \xi) d \xi d x+2 L^{2} T_{1}^{2}\right)^{n}<1$ and we conclude

$$
\begin{aligned}
& \lim _{m, n \rightarrow \infty} \sup _{0 \leq t \leq T_{1}} \sqrt{\mathbf{E}\left\|u^{(n)}(t, \cdot)-u^{(m)}(t, \cdot)\right\|_{L_{2}\left(\mathbb{R}^{d}\right)}^{2}} \\
& =\lim _{m, n \rightarrow \infty} \sup _{0 \leq t \leq T_{1}} \sqrt{\mathbf{E}\left\|\sum_{i=m-1}^{n-1}\left(u^{(i+1)}(t, \cdot)-u^{(i)}(t, \cdot)\right)\right\|_{L_{2}\left(\mathbb{R}^{d}\right)}^{2}} \\
& \leq \lim _{m, n \rightarrow \infty} \sum_{i=m-1}^{n-1} \sqrt{\sup _{0 \leq t \leq T_{1}} \mathbf{E}\left\|u^{(i+1)}(t, \cdot)-u^{(i)}(t, \cdot)\right\|_{L_{2}\left(\mathbb{R}^{d}\right)}^{2}} \\
& \leq \sqrt{\sup _{0 \leq t \leq T_{1}} \mathbf{E}\left\|u^{(1)}(t, \cdot)-u^{(0)}(t, \cdot)\right\|_{L_{2}\left(\mathbb{R}^{d}\right)}^{2}} \\
& \times \lim _{m, n \rightarrow \infty} \sum_{i=m-1}^{n-1} \sqrt{\left(2 \sup _{0 \leq t \leq T} \int_{\mathbb{R}^{d}} \int_{\mathbb{R}^{d}} l^{2}(t, x, \xi) d \xi d x+2 L^{2} T_{1}^{2}\right)^{i}}=0 .
\end{aligned}
$$

Thus, $\left(u^{(n)}(t, \cdot), n \in\{1,2, \ldots\}\right), 0<t \leq T_{1}$, is a Cauchy sequence. Consequently, there is a limiting function $u(t, \cdot) \in L_{2}\left(\mathbb{R}^{d}\right), 0<t \leq T_{1}$, such that

$$
\lim _{n \rightarrow \infty} \sup _{0 \leq t \leq T_{1}} \mathbf{E}\left\|u^{(n)}(t, \cdot)-u(t, \cdot)\right\|_{L_{2}\left(\mathbb{R}^{d}\right)}^{2}=0 .
$$

From (14), it follows from Fatou's Lemma that

$$
\sup _{0 \leq t \leq T_{1}} \mathbf{E}\|u(t, \cdot)\|_{L_{2}\left(\mathbb{R}^{d}\right)}^{2} \leq c\left(T_{1}\right) .
$$

The function $u$ is $\mathcal{F}_{t}$-measurable as a limit of $\mathcal{F}_{t}$-measurable functions.

1.3 Next we show that $u(t, \cdot), 0<t \leq T_{1}$, solves the equation (7). To this end, we need to pass to the limit in the identity (9). Taking into account (15), we have

$$
\begin{aligned}
& \lim _{n \rightarrow \infty} \sup _{0 \leq t \leq T_{1}} \mathbf{E}\left\|\int_{\mathbb{R}^{d}}\left(b\left(t, \cdot, u^{(n-1)}(\alpha(t), \xi), \xi\right)-b(t, \cdot, u(\alpha(t), \xi), \xi)\right) d \xi\right\|_{L_{2}\left(\mathbb{R}^{d}\right)}^{2} \\
& \leq\left(\sup _{0 \leq t \leq T} \iint_{\mathbb{R}^{d}} l_{\mathbb{R}^{d}}^{2}(t, x, \xi) d \xi d x\right) \lim _{n \rightarrow \infty} \sup _{0 \leq t \leq T_{1}} \mathbf{E}\left\|u^{(n-1)}(t, \cdot)-u(t, \cdot)\right\|_{L_{2}\left(\mathbb{R}^{d}\right)}^{2}=0, \\
& \lim _{n \rightarrow \infty} \sup _{0 \leq t \leq T_{1}} \mathbf{E}\left\|\int_{0}^{t}\left(f\left(s, u^{(n-1)}(\alpha(s), \cdot), \cdot\right)-f(s, u(\alpha(s), \cdot), \cdot)\right) d s\right\|_{L_{2}\left(\mathbb{R}^{d}\right)}^{2} \\
& \leq L^{2} T_{1} \lim _{n \rightarrow \infty} \int_{-r}^{\alpha\left(T_{1}\right)} \mathbf{E}\left\|u^{(n-1)}(s, \cdot)-u(s, \cdot)\right\|_{L_{2}\left(\mathbb{R}^{d}\right)}^{2} d s \\
& \leq L^{2} T_{1}^{2} \lim _{n \rightarrow \infty} \sup _{0 \leq t \leq T_{1}} \mathbf{E}\left\|u^{(n-1)}(t, \cdot)-u(t, \cdot)\right\|_{L_{2}\left(\mathbb{R}^{d}\right)}^{2}=0 .
\end{aligned}
$$


Therefore, passing to the limit in (9), we have

$$
\begin{aligned}
u(t, \cdot) & =\phi(0, \cdot)+\int_{\mathbb{R}^{d}} b(0, \cdot, \phi(-r, \xi), \xi) d \xi-\int_{\mathbb{R}^{d}} b(t, \cdot, u(\alpha(t), \xi), \xi) d \xi \\
& +\int_{0}^{t} f(s, u(\alpha(s), \cdot), \cdot) d s+\int_{0}^{t} \sigma(s, \cdot) d \beta(s), 0<t \leq T_{1},
\end{aligned}
$$

- the solution to $(7)$ on $\left[0, T_{1}\right]$. This procedure can be repeated in order to extend the solution to the entire interval $[0, T]$ in finitely many steps, thereby completing the proof.

Proof of the theorem 2. Let prove the desired result under the hypothesis M1. From now on, suppose that $x$ is fixed.

2.1 Let $u_{2}$ solve the problem

$$
\begin{gathered}
d\left(u_{2}(t, \cdot)+\int_{\mathbb{R}^{d}} b_{2}\left(t, \cdot, u_{2}(\alpha(t), \xi), \xi\right) d \xi\right)=f_{2}\left(t, u_{2}(\alpha(t), \cdot), \cdot\right) d t+\sigma(t, \cdot) d \beta(t), 0<t \leq T, \\
u_{2}(t, \cdot)=\phi_{2}(t, \cdot),-r \leq t \leq 0,
\end{gathered}
$$

i.e. satisfy the following identities

$$
\begin{gathered}
\left(u_{2}(t, \cdot)+\int_{\mathbb{R}^{d}} b_{2}\left(t, \cdot, u_{2}(\alpha(t), \xi), \xi\right) d \xi\right)-\left(u_{2}(0, \cdot)+\int_{\mathbb{R}^{d}} b_{2}\left(0, \cdot, u_{2}(\alpha(0), \xi), \xi\right) d \xi\right) \\
\quad=\int_{0}^{t} f_{2}\left(s, u_{2}(\alpha(s), \cdot), \cdot\right) d s+\int_{0}^{t} \sigma(s, \cdot) d \beta(s), 0<t \leq T \\
u_{2}(t, \cdot)=\phi_{2}(t, \cdot),-r \leq t \leq 0 .
\end{gathered}
$$

Let $u_{3}$ solve the problem

$$
\begin{gathered}
d\left(u_{3}(t, \cdot)+\int_{\mathbb{R}^{d}} b_{1}\left(t, \cdot, u_{2}(\alpha(t), \xi), \xi\right) d \xi\right)=f_{1}\left(t, u_{2}(\alpha(t), \cdot), \cdot\right) d t+\sigma(t, \cdot) d \beta(t), 0<t \leq T \\
u_{3}(t, \cdot)=\phi_{1}(t, \cdot),-r \leq t \leq 0
\end{gathered}
$$

i.e. satisfy the following identities

$$
\begin{gathered}
\left(u_{3}(t, \cdot)+\int_{\mathbb{R}^{d}} b_{1}\left(t, \cdot, u_{2}(\alpha(t), \xi), \xi\right) d \xi\right)-\left(u_{3}(0, \cdot)+\int_{\mathbb{R}^{d}} b_{1}\left(0, \cdot, u_{2}(\alpha(0), \xi), \xi\right) d \xi\right) \\
=\int_{0}^{t} f_{1}\left(s, u_{2}(\alpha(s), \cdot), \cdot\right) d s+\int_{0}^{t} \sigma(s, \cdot) d \beta(s), 0<t \leq T,
\end{gathered}
$$




$$
u_{3}(t, \cdot)=\phi_{1}(t, \cdot),-r \leq t \leq 0 .
$$

Subtracting $(17)-\left(17^{*}\right)$ from $(16)-\left(16^{*}\right)$, we obtain

$$
\begin{aligned}
& \left(u_{2}(t, \cdot)-u_{3}(t, \cdot)\right)+\underbrace{\int_{\mathbb{R}^{d}}\left(b_{2}\left(t, \cdot, u_{2}(\alpha(t), \xi), \xi\right) d \xi-b_{1}\left(t, \cdot, u_{2}(\alpha(t), \xi), \xi\right)\right) d \xi}_{\geqslant 0} \\
& +\underbrace{\left(u_{3}(0, \cdot)-u_{2}(0, \cdot)\right)}_{\geqslant 0}+\underbrace{\int_{\mathbb{R}^{d}}\left(b_{1}\left(0, \cdot, u_{2}(\alpha(0), \xi), \xi\right) d \xi-b_{2}\left(0, \cdot, u_{2}(\alpha(0), \xi), \xi\right)\right) d \xi}_{=0} \\
& =\underbrace{\int_{0}^{t}\left(f_{2}\left(s, u_{2}(\alpha(s), \cdot), \cdot\right)-f_{1}\left(s, u_{2}(\alpha(s), \cdot), \cdot\right)\right) d s}_{\leq 0}, 0<t \leq T, \\
& u_{2}(t, \cdot)-u_{3}(t, \cdot)=\underbrace{\phi_{2}(t, \cdot)-\phi_{1}(t, \cdot)}_{\leq 0},-r \leq t \leq 0,
\end{aligned}
$$

therefore $u_{2} \leq u_{3}$ with probability one.

Now let consider $u_{4}-$ a solution to

$$
\begin{gathered}
d\left(u_{4}(t, \cdot)+\int_{\mathbb{R}^{d}} b_{1}\left(t, \cdot, u_{3}(\alpha(t), \xi), \xi\right) d \xi\right)=f_{1}\left(t, u_{3}(\alpha(t), \cdot), \cdot\right) d t+\sigma(t, \cdot) d \beta(t), 0<t \leq T \\
u_{3}(t, \cdot)=\phi_{1}(t, \cdot),-r \leq t \leq 0
\end{gathered}
$$

i.e. is defined from

$$
\begin{gathered}
\left(u_{4}(t, \cdot)+\int_{\mathbb{R}^{d}} b_{1}\left(t, \cdot, u_{3}(\alpha(t), \xi), \xi\right) d \xi\right)-\left(u_{4}(0, \cdot)+\int_{\mathbb{R}^{d}} b_{1}\left(0, \cdot, u_{3}(\alpha(0), \xi), \xi\right) d \xi\right) \\
\quad=\int_{0}^{t} f_{1}\left(s, u_{3}(\alpha(s), \cdot), \cdot\right) d s+\int_{0}^{t} \sigma(s, \cdot) d \beta(s), 0<t \leq T, \\
u_{4}(t, \cdot)=\phi_{1}(t, \cdot),-r \leq t \leq 0 .
\end{gathered}
$$


Subtracting $(18)-\left(18^{*}\right)$ from $(17)-\left(17^{*}\right)$, we conclude

$$
\begin{aligned}
& \left(u_{3}(t, \cdot)-u_{4}(t, \cdot)\right)+\underbrace{\int_{\mathbb{R}^{d}}\left(b_{1}\left(t, \cdot, u_{2}(\alpha(t), \xi), \xi\right) d \xi-b_{1}\left(t, \cdot, u_{3}(\alpha(t), \xi), \xi\right)\right) d \xi}_{\geqslant 0} \\
& +\underbrace{\left(u_{4}(0, \cdot)-u_{3}(0, \cdot)\right)}_{=0}+\underbrace{\int_{\mathbb{R}^{d}}\left(b_{1}\left(0, \cdot, u_{3}(\alpha(0), \xi), \xi\right) d \xi-b_{1}\left(0, \cdot, u_{2}(\alpha(0), \xi), \xi\right)\right) d \xi}_{=0} \\
& =\underbrace{\int_{0}^{t}\left(f_{1}\left(s, u_{2}(\alpha(s), \cdot), \cdot\right)-f_{1}\left(s, u_{3}(\alpha(s), \cdot), \cdot\right)\right) d s}_{\leq 0}, 0<t \leq T, \\
& u_{3}(t, \cdot)-u_{4}(t, \cdot)=\phi_{1}(t, \cdot)-\phi_{1}(t, \cdot)=0,-r \leq t \leq 0,
\end{aligned}
$$

therefore $u_{3} \leq u_{4}$ with probability one. Continuing in a similar way, one obtain a sequence $\left(u_{n}, n \in\{2,3, \ldots\}\right)$, fulfilling

$$
u_{2} \leq u_{3} \leq u_{4} \leq \ldots \leq u_{n} \leq \ldots
$$

where $u_{n}, n \in\{5,6, \ldots\}$, is defined as

$$
\begin{gathered}
\left(u_{n}(t, \cdot)+\int_{\mathbb{R}^{d}} b_{1}\left(t, \cdot, u_{n-1}(\alpha(t), \xi), \xi\right) d \xi\right)-\left(u_{n}(0, \cdot)+\int_{\mathbb{R}^{d}} b_{1}\left(0, \cdot, u_{n-1}(\alpha(0), \xi), \xi\right) d \xi\right) \\
=\int_{0}^{t} f_{1}\left(s, u_{n-1}(\alpha(s), \cdot), \cdot\right) d s+\int_{0}^{t} \sigma(s, \cdot) d \beta(s), 0<t \leq T, \quad(19) \\
u_{n}(t, \cdot)=\phi_{1}(t, \cdot),-r \leq t \leq 0 .
\end{gathered}
$$

2.2 Hereafter we argue in a similar way as in the proof of theorem 1 . We establish that $\left(u_{n}, n \in\{2,3, \ldots\}\right)$ is convergent. In order to do it, we prove that

$$
\lim _{n \rightarrow \infty} \sup _{0 \leq t \leq T} \mathbf{E}\left\|u_{n}(t, \cdot)-u_{1}(t, \cdot)\right\|_{L_{2}\left(\mathbb{R}^{d}\right)}^{2}=0,
$$

where $u_{1}$ is defined from

$$
\begin{gathered}
\left(u_{1}(t, \cdot)+\int_{\mathbb{R}^{d}} b_{1}\left(t, \cdot, u_{1}(\alpha(t), \xi), \xi\right) d \xi\right)-\left(u_{1}(0, \cdot)+\int_{\mathbb{R}^{d}} b_{1}\left(0, \cdot, u_{1}(\alpha(0), \xi), \xi\right) d \xi\right) \\
=\int_{0}^{t} f_{1}\left(s, u_{1}(\alpha(s), \cdot), \cdot\right) d s+\int_{0}^{t} \sigma(s, \cdot) d \beta(s), 0<t \leq T \\
u_{1}(t, \cdot)=\phi_{1}(t, \cdot),-r \leq t \leq 0 .
\end{gathered}
$$

It follows from the proof of theorem 1 that there exists a constant $c(T)>0$ such that $\sup _{0 \leq t \leq T} \mathbf{E}\left\|u_{2}(t, \cdot)\right\|_{L_{2}\left(\mathbb{R}^{d}\right)}^{2} \leq c(T)$ and $\sup _{0 \leq t \leq T} \mathbf{E}\left\|u_{n}(t, \cdot)\right\|_{L_{2}\left(\mathbb{R}^{d}\right)}^{2} \leq c(T)$ for $n \in\{3,4, \ldots\}$. The rest of the proof is similar to the case of theorem 1. 


\section{Results and discussion}

As mentioned in the introduction, comparison theorems play an important role in the study of solutions with non-Lipschitz coefficients, in the study of the behavior of solutions at infinity, for optimal control of stochastic systems ([1],[2],[4],[5], [7]-[9], [11]-[25]). However, for equations with a delay of the neutral type, such studies have not been carried out before. This is due to the fact that lag is among the stochastic derivative, and therefore it is impossible to apply the classical Ito formula of differentiated functioning. Namely, on the application of Ito's formula, the proof of the classical comparison theorems is constructed.

\section{Conclusion}

Thus, the paper considers the existence, uniqueness and comparison theorems for stochastic functional-differential equations of neutral type with variable delay. When obtaining these results, the methods of stochastic and functional analysis were used, namely, the principle of compressed mappings, monotonicity methods, coupling method and others. Using these methods, we obtain local and global theorems on the existence and uniqueness of initial problems for stochastic functional differential equations with variable delay of a neutral type, as well as theorems for comparing two solutions. In the future, this method will allow us to obtain similar results for equations with unbounded operators, in particular for stochastic functional differential equations of the neutral type of partial differential equations.

\section{References}

[1] Curtain R. F., and A. J. Pritchard. "Infinite dimension linear theory systems."Lecture Notes in Control and Information Sciences, Springer-Verlag: Now York 8 (1978): 12.

[2] Gal'Cuk L. I., and M. H. A. Davis. "A note on a comparison theorem for equations with different diffusions. "Stochastics: An International Journal of Probability and Stochastic Processes 6, no. 2 (1982): 147-149.

[3] Geib C.and Manthey R. "Comparison theorems for stochastic differential equations in finite and infinite dimensions."Stochastic processes and their applications 53, no. 1 (1994): 23-35.

[4] Huang Zhi Yuan. "A comparison theorem for solutions of stochastic differential equations and its applications."Proceedings of the American Mathematical Society 91, no. 4 (1984): 611-617.

[5] Kotelenez P. "Comparison methods for a class of function valued stochastic partial differential equations."Probability Theory and related fields 93, no. 1 (1992): 1-19.

[6] Manthey R. and Zausinger T. "Stochastic evolution equations in $L^{2 \nu} \rho$."Stochastics: An International Journal of Probability and Stochastic Processes 66, no. 1-2 (1999): 37-85.

[7] O'Brien, G. L. "A new comparison theorem for solutions of stochastic differential equations."Stochastics 3, no. 1-4 (1980): 245-249.

[8] Ouknine Y. "Comparaison et non-confluence des solutions d'йquations diffŭrentielles stochastiques unidimensionnelles."Probab. Math. Statist 11 (1990): 37-46. 
[9] Tanaka H. "Stochastic differential equations"Seminar on Probability (in Japanese) 19 (1964)

[10] Yamada T. "On a comparison theorem for solutions of stochastic differential equations and its applications." Journal of Mathematics of Kyoto University 13, no. 3 (1973): 497-512.

[11] Yamada T. and Yukio O. "On the strong comparison theorems for solutions of stochastic differential equations."Zeitschrift for Wahrscheinlichkeits theorie und Verwandte Gebiete 56, no. 1 (1981): 3-19.

[12] Da Prato Giuseppe and Jerzy Zabczyk. Stochastic Equations in Infinite Dimensions. Vol. 152. Cambridge University Press, 2014.

[13] Watanabe, S. and Ikeda, N. Stokhasticheskie differencial'nye uravneniya i diffusionnye processy [Stochastic differential equations and diffusional processes].(M.:1986): Nauka, 445.

[14] Skorokhod, A. V. Issledovaniya po teorii sluchainyh processov [Research on the theory of random processes] .(Kiev: Kiev University, 1961):216

[15] Stanzhitskii A.N. and Tsukanova A.O. Sushestvovanie $i$ edinstvennost' resheniya zada Koshi dlya stohasticheskogo differencialnogouravneniya reakcii-diffuzii neitralnogo tipa [Existence and uniqueness of a solution to the Cauchy problem for a stochastic differential reaction-diffusion equation of neutral type]. Nelineinoe kolebanie. 19,№3 (2016): 408-430.

[16] Butkovsky O. "Subgeometric rates of convergence of Markov processes in the Wasserstein metric."The Annals of Applied Probability 24, no. 2 (2014): 526-552

[17] Butkovsky O. and Michael S.. "Invariant measures for stochastic functional differential equations."Electronic Journal of Probability 22 (2017).

[18] Butkovsky O., Kulik A. and Scheutzow M.. "Generalized couplings and ergodic rates for SPDEs and other Markov models."arXiv preprint arXiv:1806.00395 (2018).

[19] Doblin W.. "Elements d'une theorie generale des chaones simples constantes de Markoff."In Annales Scientifiques de l'ENS, vol. 57, pp. 61-111. 1940.

[20] Es-Sarhir, A., van Gaans, O. and Scheutzow, M. "Invariant measures for stochastic functional differential equations with superlinear drift term."Differential and Integral Equations 23, no. 1/2 (2010): 189-200.

[21] Es-Sarhir, A., Von Renesse, M.K. and Scheutzow, M.,. "Harnack inequality for functional SDEs with bounded memory."Electronic communications in probability 14 (2009): 560-565.

[22] Es-Sarhir, A., Scheutzow, M., Tolle, J.M. and van Gaans, O. "Invariant measures for monotone SPDEs with multiplicative noise term."Applied Mathematics and Optimization 68, no. 2 (2013): 275-287.

[23] Hairer M. and Mattingly J.C. "Yet another look at Harris' ergodic theorem for Markov chains."In Seminar on Stochastic Analysis, Random Fields and Applications VI, pp. 109-117. Springer, Basel, 2011.

[24] Hairer, M., Mattingly, J.C. and Scheutzow, M. "Asymptotic coupling and a general form of Harris' theorem with applications to stochastic delay equations. "Probability theory and related fields 149, no. 1-2 (2011): 223-259.

[25] Has'minskii R. Z. "Necessary and sufficient conditions for the asymptotic stability of linear stochastic systems."Theory Probability Appl 12 (1967): 144-147. 